\title{
Role of the LIV-1 subfamily of zinc transporters in the development and progression of breast cancers: A mini review
}

\author{
Tomoka Takatani-Nakase*, Chihiro Matsui and Koichi Takahashi* \\ Department of Pharmaceutics, School of Pharmacy and Pharmaceutical Sciences, Mukogawa Women's University, Japan
}

\begin{abstract}
Zinc is a trace metal essential for many cellular processes, including its recently described role as a signaling mediator. Zinc transporters regulate intracellular zinc homeostasis, control numerous physiological cell functions involved in zinc signaling, and play a role in the progression of several diseases. In breast cancer, zinc transporters are significantly involved in the epithelial mesenchymal transition and in resistance to anti-hormonal therapies, which are critical factors associated with a poor prognosis. In a previous study, we showed that the promotion of cell migration was strongly dependent on both the extracellular glucose concentration and the transport of zinc ions by zinc transporters, which play an essential role in the glucose-induced cell migration. These findings suggest that zinc transporters are key molecules involved in cellular adaptation to the extracellular environment, which has been linked to malignant progression in breast cancer. In this review, we discuss the latest findings on the pathophysiological role of zinc transporters in breast cancer and their potential as targets in breast cancer therapy.
\end{abstract}

\section{Introduction}

More than 300 molecules are required for the proper functioning of biological processes. These molecules include zinc, a trace element that acts as both a catalytic and metabolic cofactor [1]. Zinc is also required by $>2000$ transcription factors to maintain their structural integrity and regulate gene expression [1]. On the other hand, zinc was shown to play a role as a second messenger in signaling pathways linked to important physiological functions such as cell differentiation, proliferation, survival, and migration (Figure 1) [2-5]. Intracellular zinc levels are tightly regulated by zinc-binding proteins and zinc transporters [6-8]. The bi-directional transport of zinc across cell membranes is maintained by two families of proteins: ZRT IRT-like proteins (ZIPs, SLC39A) facilitate the influx of zinc into the cytosol, while zinc transporters (ZnT, SLC30A) mediate zinc efflux from the cytosol. The mammalian ZIP family contains 14 members that are divided into 4 subgroups based on the extent of sequence conservation: ZIP subfamily I (ZIP9), ZIP subfamily II (ZIP1, ZIP2, ZIP3), LIV-1 subfamily (ZIP4, ZIP5, ZIP6, ZIP7, ZIP8, ZIP10, ZIP12, ZIP13, ZIP14), and the GufA subfamily (ZIP11) [9,10]. These zinc transporters play crucial roles not only in maintaining the cellular zinc balance but also in mediating intracellular signaling pathways. Aberrations in zinc transport have been linked to diseases such as Alzheimer's disease [11], diabetes [12-14], cancer [5], and other pathologic processes [4,15,16].

The intracellular and extracellular zinc levels and distributions, controlled by zinc transporters, mediate many cell functions in disease processes. The mechanism of action of zinc in cancer depends on cancer type. In prostate cancer, low levels of zinc have been measured in serum and tumor samples, and decreased expression of ZIP1, 2, and 3 has been demonstrated [17-20]. However, in breast cancer, zinc levels are low in serum and high in tumor samples [21-24]. In addition, both clinical and in vitro findings have provided evidence of the importance of LIV-1 subfamily such as ZIP6, 7, and 10 in breast cancer progression.
These results suggest that a better understanding of the functions of zinc and ZIPs in breast cancer will aid in developing more effective therapeutic strategies $[9,10,25-29]$. In this review, we discuss current research on the role of ZIPs in breast cancer development.

\section{ZIPs and breast cancer}

\section{ZIP6 expression and the malignant progression of breast cancer}

ZIP6 is present in the plasma membrane of mammary epithelial cells and imports zinc into the cytoplasm [22]. Previous studies described an association between ZIP6, which is encoded by an estrogen-regulated gene, and breast cancer, in which estrogen plays a critical role $[9,30]$. Moreover, ZIP6 expression is tightly regulated according to the breast cancer stage. In a study on zebrafish embryos, ZIP6 expression was dependent on expression of signal transducer and activator of transcription 3 (STAT3) [31], and Hogstrand et al. [32] showed that epidermal growth factor (EGF) induces epithelial mesenchymal transition (EMT) by increasing ZIP6 expression dependency on

Correspondence to: Tomoka Takatani-Nakase, Department of Pharmaceutics, School of Pharmacy and Pharmaceutical Sciences, Mukogawa Women's University, Japan 11-68, Koshien Kyuban-cho, Nishinomiya, Hyogo 6638179, Japan, Tel: +81-798-45-9943, Fax: +81-798-45-9943; E-mail: nakase@mukogawa-u.ac.jp

Koichi Takahashi, Department of Pharmaceutics, School of Pharmacy and Pharmaceutical Sciences, Mukogawa Women's University, Japan 11-68, Koshien Kyuban-cho, Nishinomiya, Hyogo 663-8179, Japan, Tel: +81-798-45-9943, Fax +81-798-45-9943; E-mail: koichi@mukogawa-u.ac.jp

Key words: zinc transporters, breast cancer, epithelial mesenchymal transition, cancer cel migration, extracellular environment

Received: May 10, 2016; Accepted: June 17, 2016; Published: June 20, 2016 


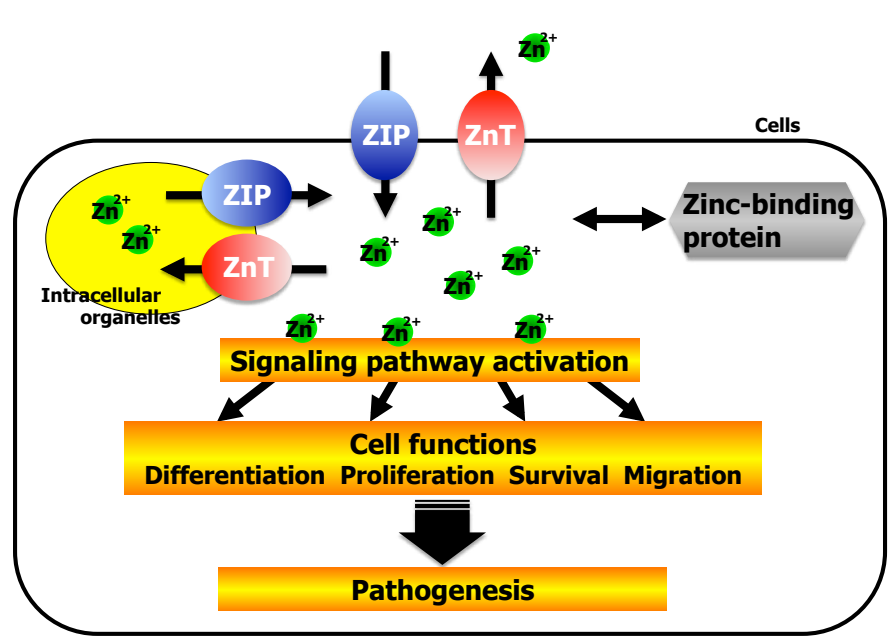

Figure 1. Cell functions and pathogenesis regulated by zinc transporters.Strategies for subtypes--dealing with the diversity of breast cancer: highlights of 19 the St. Gallen International Expert Consensus on the Primary Therapy of Early Breast Cancer.

STAT3. The EMT is considered to be an essential feature of malignancy, and during this transition, cellular adhesion is lost, allowing cells to migrate. ZIP6 mediates cell detachment, which allows cells to migrate and become metastatic. This result corroborates with the high levels of ZIP6 found in estrogen receptor-positive breast cancer patients with lymph node metastasis $[9,10,26]$. While these findings signify pathological roles for ZIP6, in some settings ZIP6 can have protective (i.e., tumor-constraining) roles; the knockdown of ZIP6 in adherent estrogen-receptor positive breast cancer cells, MCF-7 cells and in T47D human ductal breast epithelial tumor cells, causes apoptosis resistance and tumorigenesis and promotes the EMT $[33,34]$. Furthermore, there is an association of LIV-1 expression with less aggressive tumours due to high E-cadherin expression $[32,35]$. Taken together, these data support previous clinical reports on the negative correlation between ZIP6 expression and tumor grade, size, and stage [30]. They also demonstrate that the role of ZIP6 differs in metastatic foci compared with that in the primary tumor. A detailed understanding of cellular zinc dynamics and the signaling pathways involved in the response to ZIP6 expression is needed to fully understand the role played by ZIP6 in breast cancer.

\section{Role of ZIP 10 in the migration of breast cancer cells}

An association between ZIP10, the family member closest to ZIP6, and the invasion and metastasis of human breast cancer cells was reported by Kagara et al. [29], who quantified the expression of ZIP10 mRNA in 177 surgical samples derived from breast cancer patients. Using real-time quantitative PCR, the authors demonstrated that the mRNA expression of ZIP10 in the breast cancer tissues of patients with lymph node metastasis was significantly higher than that in patients without metastasis [29].

Similarly, ZIP10 mRNA was more highly expressed in a metastatic than in a non-metastatic breast cancer cell line. ZIP10-knockdown in the metastatic cell line decreased both zinc intake and cell migration. These findings support essential roles for zinc and ZIP10 in the migratory activity of highly metastatic breast cancer cells.

\section{Role of ZIP7 in tamoxifen-resistant breast cancer}

Upon stimulation, normal cells exhibit "early" and "late" events [22]. Early zinc signaling originates from the endoplasmic reticulum (ER) and is observed several minutes after stimulation. Then the released zinc can modulates the activities of intracellular signaling molecules. ZIP7, which is located in the ER membrane, mediates this early zinc influx. Phosphorylation by casein kinase II (CK2), an enzyme that promotes cell division, is required for ZIP7 activation and results in the release of zinc ions from the ER into the cytoplasm. The released zinc ions inhibit protein phosphatases and enhance the activity of tyrosine kinases as well as the Akt survival and ERK1/2 growth signaling pathways, which together increase cancer progression (Figure 2) [26]. ZIP7 is abundantly expressed in tamoxifen-resistant MCF-7 breast cancer cells, where it is located in the ER [28]. Since ZIP7 activation by CK2 enhances the proliferation and migration of breast cancer cells, it may be a promising and novel molecular target for breast cancer chemotherapy $[25,26]$. However, several questions remain to be answered: are the above-described observations limited to tamoxifenresistant breast cancer cells? Are these results mimicked in vivo? Are other zinc transporters besides ZIP7 involved? Is the release of zinc ion from the ER dependent on ZIP7 activity? One challenge in answering these questions is that free zinc levels in the ER and elsewhere in the cell vary greatly depending on the detection assay used (zinc smallmolecule sensors, genetically encoded fluorescence resonance energy transfer [FRET] sensors), although consistent results have generally been reported for cytosolic levels [36].

High glucose levels contribute to the essential roles of ZIP6 and ZIP10 in the promotion of cell motility

Breast cancer patients who are also diabetic have an even higher risk of mortality, and both the therapeutic regimen and its observed effects differ between patients with and without diabetes [37-40]. These findings suggest that the currently established strategies for breast cancer therapy may not be suitable for diabetic patients. A better understanding of the relationship between breast cancer and diabetes would improve clinical approaches and methodologies. The extracellular environment of cancer cells influences their growth and behavior, with effects documented for invasion, metastasis, and tumor development [41]. In previous work, we demonstrated that

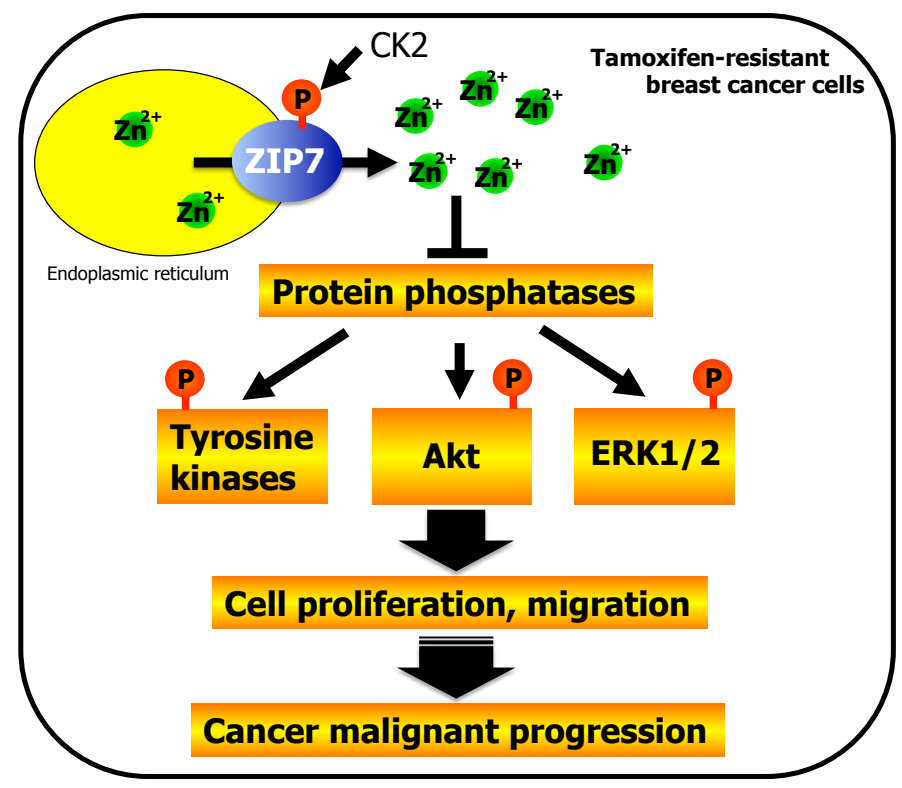

Figure 2. ZIP7-mediated zinc signaling in tamoxifen-resistant breast cancer cells. ZIP7 is located in the endoplasmic reticulum and is highly expressed in tamoxifen-resistant breast cancer cells. ZIP7-mediated zinc signaling contributes to cell proliferation and migration; these are characteristic features of anti-hormone-resistant cancers. 
MCF-7 breast cancer cells cultured in medium containing glucose levels corresponding to a hyperglycemic state have a significantly higher motility than cells exposed to physiological glucose levels [42]. Moreover, ZIP6 and ZIP10 expression levels are elevated in cells exposed to high versus physiological glucose levels. The depletion of intracellular zinc ions by zinc chelation and the knockdown of ZIP6 or ZIP10 prevents the enhancement of cell migration, indicating that the transport of zinc ions by ZIP6 and ZIP10 plays an essential role in cell motility stimulated by high glucose levels [43].

In cancer cells, both the molecular species and the expression levels of zinc transporters vary depending on the extracellular environment. An increase in ZIP6 expression is required for the metastatic transformation of MCF-7 cells [32], and ZIP10 mRNA levels are significantly higher in breast cancer patients who have lymph node metastasis than in those who do not [29]. Together with the abovementioned observations on the roles of ZIP6 and ZIP10 in enhancing cancer cell migration in response to high blood glucose [42], these finding support studies on ZIP6 and ZIP10 as candidate markers of metastatic spread in breast cancer patients with diabetes-related hyperglycemia. Taylor et al. [28] showed that in anti-hormone resistant cells the uptake of intracellular zinc ions plays an essential role in cell migratory activity, a result confirmed in our laboratory. Since ZIP7 is expressed in the ER but not in the plasma membrane in MCF-7 cells, breast cancer cell migration and metastasis may require intracellular zinc ion uptake by plasma membrane-located ZIP6 and ZIP10 and by ER-localized ZIP7. However, the relationship between ZIP7 expression and lymph node metastasis remains to be demonstrated in clinical studies.

\section{Potential role for ZIPs in anticancer drug activity}

The pathways controlling cell survival and death and the acquisition of apoptosis resistance are likely to be advantageous targets in breast cancer therapy. Autophagy, in which autophagosomes form around misfolded proteins and damaged organelles to induce their degradation, is crucial for not only maintaining cell homeostasis but also controlling cell death, it has also been implicated in the development of breast cancer $[43,44]$. In MCF-7 cells, intracellular zinc ions accumulate in autophagosomes and are required for autophagosome-mediated tamoxifen-induced cell death [45]. The recent demonstration of the zinc transporters ZIP4, ZIP14, ZIP8, ZnT10, ZnT4, and ZnT2 in endosomes, lysosomes, and autolysosomes suggests that zinc is an essential contributor to autophagy $[46,47]$. Investigations into the roles of zinc and zinc transporters in breast cancer have recently began, and will need to take into account the relationships between the LIV-1 subfamily of zinc transporters and autophagy.

Studies on colorectal cancer have shown that the antipyrimidine 5-fluorouracil (5-FU) increases the intracellular concentration of labile zinc, resulting in growth inhibition through the activation of p53 and the repression of NF- $\kappa B$ [48]. The addition of zinc enhances growth inhibition by 5 -FU, and zinc re-sensitizes 5 -FU-resistant cell lines to the drug, such that their response is comparable to that of sensitive cell lines [48]. Moreover, in a xenograft model of wild type p53carrying colon cancer, it was reported that low-dose adriamycin did not induce tumor regression unless it was given in combination with zinc chloride $\left(\mathrm{ZnCl}_{2}\right)$, because $\mathrm{ZnCl} 2$ activated endogenous wild- type p53 and transactivated target genes in response to low drug dose [49]. Blanden et al. [50] showed that synthetic zinc metallochaperone-1 (ZMC1) increased the level of intracellular labile zinc ions by shuttling extracellular zinc across the plasma membrane, $\mathrm{ZMC1}$ rescued the mutant p53 phenotype, suggesting that it might be a valuable tool for in the development of anticancer drugs targeting mutant p53. These reports show that the addition of zinc has a positive effect on anticancer therapies. Abnormal p53 expression is the most frequent genetic alteration in breast cancers (approximately 30\%) [51]. To determine if a combination strategy (zinc and anticancer drugs) would aid the treatment of breast cancer, additional studies are needed on the effects of zinc transport into cells, p53 status, and the mechanism by which ZIPs regulate intracellular labile zinc levels in the context of chemotherapies.

\section{Conclusion and future challenges}

In-depth studies on the role of zinc ions and ZIPs in breast cancer are likely to reveal novel functions for zinc, as well as promising strategies to enhance the efficacy of chemotherapy. This review has discussed several critical roles of zinc and its transporters in breast cancer.

Breast cancer is a complex and heterogeneous disease. Thus far, five different types have been recognized based on histology, tumor grade, growth rate, lymph node metastasis, and the presence of predictive markers, such as the estrogen and progesterone receptors and the human epidermal growth factor receptor 2 (HER2) [52-54]. Tumor typing guides the selection of effective anti-cancer therapies based on the molecular biology of the tumor. A better understanding of the effects and roles of ZIPs and zinc signaling in breast cancer may contribute to more accurate diagnoses and more effective therapeutic strategies.

Several questions remain regarding the mechanisms underlying the regulation of gene expression by ZIPs, the zinc-sensing mechanism of cells, the roles of ZIPs and ZnTs in the zinc cellular network and the control of zinc reservoirs (including intracellular metallothionein or glutathione), and the reservoirs of other metal ions in breast cancer. These studies will be aided by the use of ZIP-knockout mice, ZIPknockdown breast cancer cell models, and cells with conditional ZIP expression, and the results will improve our understanding of the mechanisms underlying breast cancer development. Detailed analyses of zinc dynamics and zinc signaling require adequate and sensitive tools, such as the fluorescent chelating probes currently used as zinc sensors in analyses of intracellular free zinc levels. However, the applicability of these and many other probes is limited by challenges in recognizing their subcellular localization, their poor zinc sensitivity, and their low dynamic range due to proton interference [55]. Recently, Hessels et al. [36] demonstrated the localization of two FRET-based $\mathrm{Zn}^{2+}$ sensors in the cytosol and ER of MCF-7 and tamoxifen-resistant MCF-7 cells. In addition to revealing the cellular localization of zinc, these probes can aid in elucidating novel biochemical functions of zinc ions and their transporters within the cellular network.

\section{Acknowledgements}

We thank Ms. Satomi Kawahara and Ms. Sachie Maeda (Department of Pharmaceutics, School of Pharmacy and Pharmaceutical Sciences, Mukogawa Women's University) for their dedicated support. Our own studies described herein were supported by a Grant-in-Aid for Scientific Research from the Japan Society for the Promotion of Science (15K07955 to T. T-N.).

\section{References}

1. Chasapis CT, Loutsidou AC, Spiliopoulou CA, Stefanidou ME (2012) Zinc and human health: an update. Arch Toxicol 86: 521-534. [Crossref] 
2. Fukada T, Yamasaki S, Nishida K, Murakami M, Hirano T (2011) Zinc homeostasis and signaling in health and diseases: Zinc signaling. J Biol Inorg Chem 16: 1123-1134. [Crossref]

3. Kambe T, Hashimoto A, Fujimoto S (2014) Current understanding of ZIP and ZnT zinc transporters in human health and diseases. Cell Mol Life Sci 71: 3281-3295. [Crossref]

4. Fukada T, Kambe T (2011) Molecular and genetic features of zinc transporters in physiology and pathogenesis. Metallomics 3: 662-674. [Crossref]

5. Murakami M, Hirano $\mathrm{T}$ (2008) Intracellular zinc homeostasis and zinc signaling. Cancer Sci 99: 1515-1522. [Crossref]

6. Kambe T, Tsuji T, Hashimoto A, Itsumura N (2015) The Physiological, Biochemical, and Molecular Roles of Zinc Transporters in Zinc Homeostasis and Metabolism. Physiol Rev 95: 749-784. [Crossref]

7. Maret W (2008) Metallothionein redox biology in the cytoprotective and cytotoxic functions of zinc. Exp Gerontol 43: 363-369. [Crossref]

8. Outten CE, O'Halloran TV (2001) Femtomolar sensitivity of metalloregulatory proteins controlling zinc homeostasis. Science 292: 2488-2492. [Crossref]

9. Taylor KM, Morgan HE, Smart K, Zahari NM, Pumford S, et al. (2007) The emerging role of the LIV-1 subfamily of zinc transporters in breast cancer. Mol Med 13: 396-406. [Crossref]

10. Taylor KM, Nicholson RI (2003) The LZT proteins; the LIV-1 subfamily of zinc transporters. Biochim Biophys Acta 1611: 16-30. [Crossref]

11. Lyubartseva G, Smith JL, Markesbery WR, Lovell MA (2010) Alterations of zinc transporter proteins ZnT-1, ZnT-4 and ZnT-6 in preclinical Alzheimer's disease brain. Brain Pathol 20: 343-350. [Crossref]

12. Sladek R, Rocheleau G, Rung J, Dina C, Shen L, et al. (2007) A genome-wide association study identifies novel risk loci for type 2 diabetes. Nature 445: 881-885. [Crossref]

13. Wenzlau JM, Juhl K, Yu L, Moua O, Sarkar SA, et al. (2007) The cation efflux transporter ZnT8 (Slc30A8) is a major autoantigen in human type 1 diabetes. Proc Natl Acad Sci U S A 104: 17040-17045. [Crossref]

14. Chimienti F, Devergnas S, Favier A, Seve M (2004) Identification and cloning of a betacell-specific zinc transporter, ZnT-8, localized into insulin secretory granules. Diabetes 53: 2330-2337. [Crossref]

15. Fukada T, Yamasaki S, Nishida K, Murakami M, Hirano T (2011) Zinc homeostasis and signaling in health and diseases: Zinc signaling. J Biol Inorg Chem 16: 1123-1134. [Crossref]

16. Wang K, Zhou B, Kuo YM, Zemansky J, Gitschier J (2002) A novel member of a zinc transporter family is defective in acrodermatitis enteropathica. Am J Hum Genet 71: 66-73. [Crossref]

17. Rishi I, Baidouri H, Abbasi JA, Bullard-Dillard R, Kajdacsy-Balla A, et al. (2003) Prostate cancer in African American men is associated with downregulation of zinc transporters. Appl Immunohistochem Mol Morphol 11: 253-260. [Crossref]

18. Franklin RB, Feng P, Milon B, Desouki MM, Singh KK, et al. (2005) hZIP1 zinc uptake transporter down regulation and zinc depletion in prostate cancer. Mol Cancer 4: 32. [Crossref]

19. Desouki MM, Geradts J, Milon B, Franklin RB, Costello LC (2007) hZip2 and hZip3 zinc transporters are down regulated in human prostate adenocarcinomatous glands. Mol Cancer 6: 37. [Crossref]

20. Kolenko V, Teper E, Kutikov A, Uzzo R (2013) Zinc and zinc transporters in prostate carcinogenesis. Nat Rev Urol 10: 219-226. [Crossref]

21. Gumulec J, Masarik M, Krizkova S, Adam V, Hubalek J, et al. (2011) Insight to physiology and pathology of zinc(II) ions and their actions in breast and prostate carcinoma. Curr Med Chem 18: 5041-5051. [Crossref]

22. Alam S, Kelleher SL (2012) Cellular mechanisms of zinc dysregulation: a perspective on zinc homeostasis as an etiological factor in the development and progression of breast cancer. Nutrients 4: 875-903. [Crossref]

23. Tinoco-Veras CM, Bezerra Sousa MS, da Silva BB, Franciscato Cozzolino SM, Viana Pires L, et al. (2011) Analysis of plasma and erythrocyte zinc levels in premenopausal women with breast cancer. Nutr Hosp 26: 293-297. [Crossref]

24. Larner F, Woodley LN, Shousha S, Moyes A, Humphreys-Williams E, et al. (2015) Zinc isotopic compositions of breast cancer tissue. Metallomics 7: 112-117. [Crossref]
25. Hogstrand C, Kille P, Nicholson RI, Taylor KM (2009) Zinc transporters and cancer: a potential role for ZIP7 as a hub for tyrosine kinase activation. Trends Mol Med 15: 101-111. [Crossref]

26. Taylor KM, Hiscox S, Nicholson RI, Hogstrand C, Kille P (2012) Protein kinase CK2 triggers cytosolic zinc signaling pathways by phosphorylation of zinc channel ZIP7. Sci Signal 5: ra11. [Crossref]

27. Manning DL, Robertson JF, Ellis IO, Elston CW, McClelland RA, et al. (1994) Oestrogen-regulated genes in breast cancer: association of pLIV1 with lymph node involvement. Eur J Cancer 30A: 675-678. [Crossref]

28. Taylor KM, Vichova P, Jordan N, Hiscox S, Hendley R, et al. (2008) ZIP7-mediated intracellular zinc transport contributes to aberrant growth factor signaling in antihormone-resistant breast cancer cells. Endocrinology 149: 4912-4920. [Crossref]

29. Kagara N, Tanaka N, Noguchi S, Hirano T (2007) Zinc and its transporter ZIP10 are involved in invasive behavior of breast cancer cells. Cancer Sci 98: 692-697. [Crossref]

30. Kasper G, Weiser AA, Rump A, Sparbier K, Dahl E, et al. (2005) Expression levels of the putative zinc transporter LIV-1 are associated with a better outcome of breast cancer patients. Int J Cancer 117: 961-973. [Crossref]

31. Yamashita S, Miyagi C, Fukada T, Kagara N, Che YS, et al. (2004) Zinc transporter LIVI controls epithelial-mesenchymal transition in zebrafish gastrula organizer. Nature 429: 298-302. [Crossref]

32. Hogstrand C, Kille P, Ackland ML, Hiscox S, Taylor KM (2013) A mechanism for epithelial-mesenchymal transition and anoikis resistance in breast cancer triggered by zinc channel ZIP6 and STAT3 (signal transducer and activator of transcription 3). Biochem J 455: 229-237. [Crossref]

33. Lopez V, Kelleher SL (2010) Zip6-attenuation promotes epithelial-to-mesenchyma transition in ductal breast tumor (T47D) cells. Exp Cell Res, 316: 366-375. [Crossref]

34. Ma X, Ma Q, Liu J, Tian Y, Wang B, et al. (2009) Identification of LIV1, a putative zinc transporter gene responsible for HDACi-induced apoptosis, using a functional gene screen approach. Mol Cancer Ther 8: 3108-3116. [Crossref]

35. Shen H, Qin H, Guo J (2009) Concordant correlation of LIV-1 and E-cadherin expression in human breast cancer cell MCF-7. Mol Biol Rep 36: 653-659. [Crossref]

36. Hessels AM, Taylor KM, Merkx M (2016) Monitoring cytosolic and ER Zn(2+) in stimulated breast cancer cells using genetically encoded FRET sensors. Metallomics 8: 211-217. [Crossref]

37. Peairs KS, Barone BB, Snyder CF, Yeh HC, Stein KB, et al. (2011) Diabetes mellitus and breast cancer outcomes: a systematic review and meta-analysis. J Clin Oncol 29: 40-46. [Crossref]

38. Srokowski TP, Fang S, Hortobagyi GN, Giordano SH (2009) Impact of diabetes mellitus on complications and outcomes of adjuvant chemotherapy in older patients with breast cancer. J Clin Oncol 27: 2170-2176. [Crossref]

39. Fleming ST, Pursley HG, Newman B, Pavlov D, Chen K (2005) Comorbidity as a predictor of stage of illness for patients with breast cancer. Med Care 43: 132-140. [Crossref]

40. van de Poll-Franse LV, Houterman S, Janssen-Heijnen ML, Dercksen MW, Coebergh JW, et al. (2007) Less aggressive treatment and worse overall survival in cancer patients with diabetes: a large population based analysis. Int J Cancer 120: 1986-1992. [Crossref]

41. Takatani-Nakase T (2013) Migration behavior of breast cancer cells in the environmen of high glucose level and the role of zinc and its transporter. Yakugaku Zasshi 133: 1195-1199. [Crossref]

42. Takatani-Nakase T, Matsui C, Maeda S, Kawahara S, Takahashi K (2014) High glucose level promotes migration behavior of breast cancer cells through zinc and its transporters. PLoS One 9: e90136. [Crossref]

43. Wang C, Hu Q, Shen HM (2016) Pharmacological inhibitors of autophagy as nove cancer therapeutic agents. Pharmacol Res 105: 164-175. [Crossref]

44. Lindqvist LM, Simon AK, Baehrecke EH (2015) Current questions and possible controversies in autophagy. Cell Death Discov 1. [Crossref]

45. Hwang JJ, Kim HN, Kim J, Cho DH, Kim MJ, et al. (2010) Zinc(II) ion mediates tamoxifen-induced autophagy and cell death in MCF-7 breast cancer cell line. Biometals 23: 997-1013. [Crossref]

46. Lopez V, Foolad F, Kelleher SL (2011) ZnT2-overexpression represses the cytotoxic effects of zinc hyper-accumulation in malignant metallothionein-null T47D breast tumor cells. Cancer Lett 304: 41-51. [Crossref] 
47. Liuzzi JP, Guo L, Yoo C, Stewart TS (2014) Zinc and autophagy. Biometals 27: 10871096. [Crossref]

48. Arriaga JM, Greco A, Mordoh J, Bianchini M (2014) Metallothionein 1G and zinc sensitize human colorectal cancer cells to chemotherapy. Mol Cancer Ther 13: 13691381. [Crossref]

49. Garufi A, Ubertini V, Mancini F, D’Orazi V, Baldari S, et al. (2015) The beneficial effect of Zinc(II) on low-dose chemotherapeutic sensitivity involves $\mathrm{p} 53$ activation in wildtype p53-carrying colorectal cancer cells. J Exp Clin Cancer Res 34: 87. [Crossref]

50. Blanden AR, Yu X, Wolfe AJ, Gilleran JA, Augeri DJ, et al. (2015) Synthetic metallochaperone $\mathrm{ZMC1}$ rescues mutant 53 conformation by transporting zinc into cells as an ionophore. Mol Pharmacol 87: 825-831. [Crossref]

51. Dumay A, Feugeas JP, Wittmer E, Lehmann-Che J, Bertheau P, et al. (2013) Distinct tumor protein p53 mutants in breast cancer subgroups. Int J Cancer 132: 1227-1231. [Crossref]

52. Holliday DL, Speirs V (2011) Choosing the right cell line for breast cancer research Breast Cancer Res 13: 215. [Crossref]

53. Goldhirsch A, Wood WC, Coates AS, Gelber RD, Thurlimann B, et al. (2011) Strategies for subtypes--dealing with the diversity of breast cancer: highlights of 19 the St. Gallen International Expert Consensus on the Primary Therapy of Early Breast Cancer. Ann Oncol 22: 1736-1747.

54. Foulkes WD, Smith IE, Reis-Filho JS (2010) Triple-negative breast cancer. $N$ Engl $J$ Med 363: 1938-1948. [Crossref]

55. Maret W (2015) Analyzing free zinc(II) ion concentrations in cell biology with fluorescent chelating molecules. Metallomics 7: 202-211. [Crossref]

Copyright: (C2016 Takatani-Nakase T. This is an open-access article distributed under the terms of the Creative Commons Attribution License, which permits unrestricted use, distribution, and reproduction in any medium, provided the original author and source are credited. 